\title{
Discovery of the first young brown dwarf in the Serpens cluster ${ }^{\star}$
}

\author{
N. Lodieu ${ }^{1}$, E. Caux ${ }^{2}$, J.-L. Monin ${ }^{3,4}$, and A. Klotz ${ }^{2}$ \\ 1 Astrophysikalisches Institut Potsdam, An der Sternwarte 16, 14482 Potsdam, Germany \\ 2 CESR CNRS-UPS, BP 4346, 31028 Toulouse Cedex 04, France \\ ${ }^{3}$ Laboratoire d'Astrophysique, Observatoire de Grenoble, BP 53, 38041 Grenoble, Cedex 09, France \\ 4 Institut Universitaire de France
}

Received 10 December 2001 / Accepted 8 January 2002

\begin{abstract}
In this letter, we report the discovery of the first young early L-dwarf in the Serpens cloud. It is obscured by more than ten magnitudes of visual absorption and was found during a near infrared (NIR) photometric survey of a 50 square arcmin area in the Serpens cloud, deep enough to probe the substellar domain. After selection from NIR colour-magnitude and colour-colour diagrams, its substellar nature has been confirmed by NIR spectroscopy at the VLT. We estimate the mass of this brown dwarf to be $\sim 0.05 M_{\odot}$ and its age to be $\sim 3.5 \mathrm{Myr}$. From its NIR indices, we estimate its present spectral type to be L0-L3, and using a model its future spectral type to be $T$. This is the first young brown dwarf ever found deeply embedded in a star formation region.
\end{abstract}

Key words. stars: formation - stars: pre-main sequence - stars: low-mass, brown dwarfs - infrared: stars techniques: photometric - techniques: spectroscopic

\section{Introduction}

Brown dwarfs are no longer elusive objects. In the recent years, a growing number of brown dwarfs have been found (see Basri 2000 and references therein). More recently, new studies have focussed on the search of young brown dwarfs in star-forming regions (Martin et al. 2001a; Martin et al. 2001b; Lucas \& Roche 2001; Béjar et al. 2001; Lucas et al. 2001). These regions are well suited locations to search for substellar objects because when young (a few Myr), lowmass stars and brown dwarfs are precisely at a stage when they are much more luminous. In the NIR range, where the bulk of the energy of such stars is emitted, this translates in a few magnitudes brighter. Therefore, through 10 mag of visual absorption $\left(A_{K} \sim 1\right)$, such young brown dwarfs can be easily detected by NIR deep observations.

The identification of a significant number of young "pre Main Sequence" brown dwarfs in a well studied star formation region will allow to constrain the IMF (Initial Mass Function) down to the substellar regime and will help us answer to fundamental questions relative to star formation: is the IMF universal? Does it keep rising in the substellar regime? How is the brown dwarf formation

Send offprint requests to: N. Lodieu,

e-mail: nlodieu@aip.de

* Based on observations collected with NTT/SOFI and VLT/ISAAC instruments at the European Southern Observatories, La Silla and Paranal, Chile (ESO Programmes P63.L-0227 and P65.L-0637). process connected to the one of solar type stars? Do the brown dwarfs present the same spatial distribution than normal stars in the cloud? Deep photometric and spectroscopic observations can help to answer these questions.

In search for this young substellar population, we have performed a very deep photometric NIR survey $\left(M_{K}=\right.$ 18.6) in the Serpens cluster, one of the nearest active star forming regions in our Galaxy (Kaas 1999). It lies in the Galactic disk $260 \pm 37 \mathrm{pc}$ from the Sun (Straizys et al. 1996). From this survey, and using recent brown dwarf models (Baraffe et al. 1998), we have selected the best brown dwarf candidates to observe them using the NIR spectrometer ISAAC on the VLT. Detailed results from the photometric survey and the method used to select all the brown dwarf candidates (from colour-magnitude and colour-colour diagrams) for subsequent spectroscopic observations with the VLT, will be presented in detail in a companion paper (Lodieu et al. 2002b). In this letter we report the discovery of the first young $(\sim 3.5 \mathrm{Myr})$ brown dwarf associated with the Serpens cluster.

\section{Observations and data analysis}

\subsection{NTT/SOFI NIR photometric observations}

The photometric observations were carried out with SOFI in the $J, H$ and $K s$ photometric bands at the ESO 3.5meter NTT in La Silla. The NIR camera SOFI is equipped with a $1024 \times 1024 \mathrm{HgCdTe}(\mathrm{NICMOS}$ III) array cooled 
Table 1. Log of the NTT/SOFI photometric observations performed on July 25, 26 and 27, 1999, translated to the JHK standard system.

\begin{tabular}{ccccc}
\hline Band & $\begin{array}{c}\text { Seeing } \\
\left({ }^{\prime \prime}\right)\end{array}$ & $N_{\text {exp }}$ & $\begin{array}{c}\text { Int. time } \\
(\min )\end{array}$ & $\begin{array}{c}\text { BD-Ser 1 } \\
\text { magnitude }\end{array}$ \\
\hline$J$ & 1.1 & 10 & 50 & $17.32( \pm 0.05)$ \\
$H$ & 1.0 & 9 & 45 & $15.57( \pm 0.05)$ \\
$K$ & 0.9 & 6 & 15 & $14.54( \pm 0.05)$ \\
\hline
\end{tabular}

at $77 \mathrm{~K}$ by liquid nitrogen (Moorwood \& Spyromilio 1997). We used the Large Field mode covering $4.94^{\prime} \times 4.94^{\prime}$ with a spatial sampling of $0.29^{\prime \prime} /$ pixel.

Two adjacent fields in the Serpens cloud were observed. Each field was recorded with 5 series of $N_{\exp } 60 \mathrm{~s}$ exposures in $J$ and $H$ and $30 \mathrm{~s}$ in $K s$ (see Table 1 for a journal of the observations). Every exposure was offseted by about $20^{\prime \prime}$ from the previous one to allow removal of sky background and eventual defaults like cosmic rays by subsequent image processing using the jitter package in the ECLIPSE software (Devillard 1999). Dark frames were recorded using the same integration times as the target field frames, and flat fields were measured during dusk and dawn before and after the observations. Standard photometric stars (Persson et al. 1998) were systematically observed before and after each series of exposures. All frames in a given band were coadded to provide final images with total integration times given in Table 1. Subsequent aperture photometry was performed with the DAOPHOT package in $\operatorname{IRAF}^{1}$, using a constant PSF across the FOV, a reasonnable assumption for SOFI Large Field observations. Results were translated from instrumental magnitudes to the standard JHK system using the calibration stars measurements and checking the magnitudes of the brightest stars against previous studies (Eiroa \& Casali 1992; Giovannetti et al. 1998). The bulk of the photometric uncertainties quoted in Table 1 is coming from this translation. Following the UAI standards, we named this new brown dwarf candidate ESOBD-Ser J182953.1+011233, short name BD-Ser 1.

\subsection{VLT/ISAAC NIR spectroscopic observations}

From the NTT/SOFI photometric observations, a set of 20 brown dwarf candidates were selected for further spectroscopic investigations. In this section, we only present the observations concerning the newly confirmed brown dwarf BD-Ser 1, which was found to lie along a reddening vector of $A_{V}=10.7$ from the dwarf end of the mainsequence locus in the colour-colour diagram.

${ }^{1}$ IRAF is distributed by National Optical Astronomy Observatories, which is operated by the Association of Universities for Research in Astronomy, Inc., under contract with the National Science Foundation.
Table 2. Log of the VLT/ISAAC spectroscopic observations performed on July 17, 2000.

\begin{tabular}{cccc}
\hline Filter & $\begin{array}{c}\text { Seeing } \\
\left({ }^{\prime \prime}\right)\end{array}$ & $N_{\text {exp }}$ & $\begin{array}{c}\text { Integration time } \\
(\min )\end{array}$ \\
\hline$J$ & 0.84 & 48 & 60 \\
$S H$ & 0.74 & 12 & 15 \\
$S K$ & 0.81 & 8 & 10 \\
\hline
\end{tabular}

These observations were carried out at the ESO 8.2-meter Very Large Telescope Unit 1 (VLT Antu) on Paranal with the short wavelength (SW) channel of the NIR Spectrometer and Array Camera ISAAC (Moorwood \& Spyromilio 1997). The short wavelength camera is equipped with a Rockwell Hawaii $1024 \times 1024 \mathrm{HgCdTe}$ array. We used the SW long slit low-resolution mode and the $J, S H$ and $S K$ ESO spectrometric filters $\left(R_{\lambda} \sim 500\right.$ for a $1^{\prime \prime}$ slit $)$.

In each spectral band, a series of $N_{\exp } 75 \mathrm{~s}$ exposures were obtained, in order to reach a Signal to Noise Ratio (SNR) of 10 accross the whole band (see Table 2 for a journal of the observations). This SNR was not achieved due to unfavourable weather conditions. Dark frames were also recorded with the same integration times, and flat fields were measured during dusk and dawn before and after the observations. Wavelength calibration was obtained using Ar and Ne arc lamp illumination recorded at the beginning of every night. Standard spectroscopic stars (Pickles 1998) were systematically observed before and after each series of exposures. The initial data processing was carried out using the ECLIPSE processing software package (Devillard 2001). Sky background was removed and further data reduction operations were applied to correct for misalignment of the slit on the detector and optical aberrations in the instrument. The resulting composite spectrum of BD-Ser 1 was obtained after airmass and absorption correction and cross calibration of the $J, S H$ and $S K$ spectral bands; it is presented in Fig. 2.

\section{Results and discussion}

\subsection{Photometry}

The selection of BD-Ser 1 for subsequent spectroscopic observations was made essentially from its position in NIR colour-magnitude and colour-colour diagrams, using evolutionary tracks computed for young $(\leq 10 \mathrm{Myr})$ brown dwarfs by Baraffe et al. (1998). Figure 1 presents the location of BD-Ser 1 in a dereddened absolute colourmagnitude diagram. The photometry of BD-Ser 1 is compatible with that of a $0.05 M_{\odot}, 3.5 \mathrm{Myr}$ old deeply embedded $\left(A_{V}=10.7\right)$ brown dwarf. However, the $J-K$ error bars prevents a precise estimation of its masse and age. We have therefore used supplementary information from its spectrum to improve this estimation. 


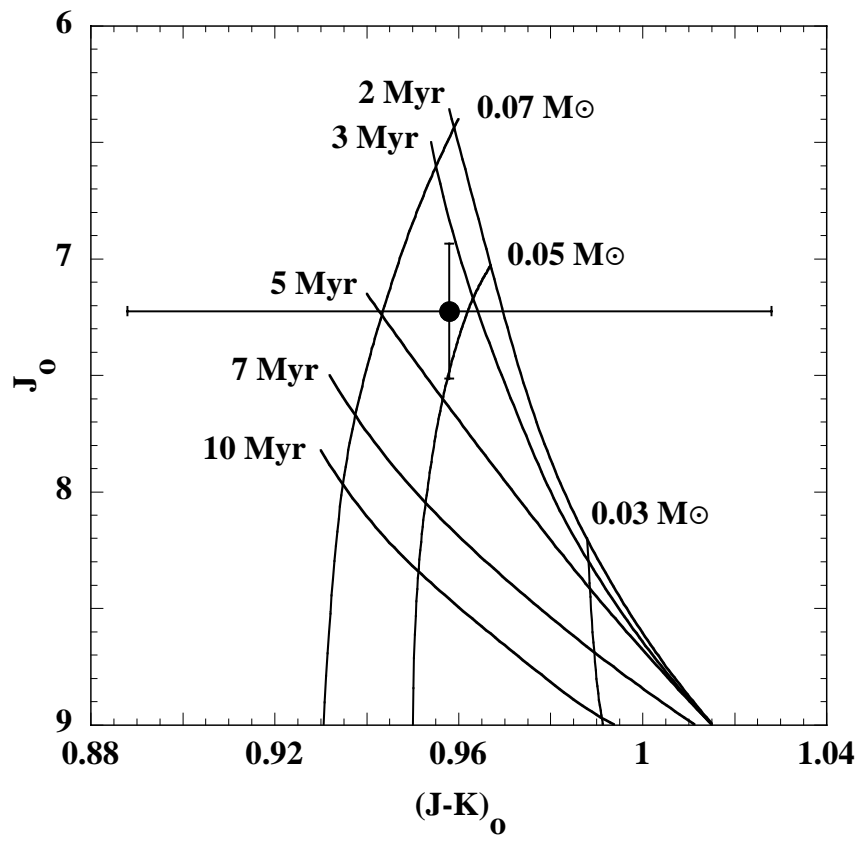

Fig. 1. Location of BD-Ser 1 in a dereddened absolute magnitude-colour diagram. Substellar isochrones and isomasses are adapted from Barraffe et al. (1998). The $J-K$ error bars are from the magnitudes uncertainties, those on $J_{0}$ are mainly due to the uncertainty on the distance to the Serpens cluster.

\subsection{Spectroscopy}

We have checked the spectrum of BD-Ser 1 for typical brown dwarf features as the $\mathrm{CO}$ bands at $2.3-2.38 \mu \mathrm{m}$ and the water absorption bands at $1.11-1.16 \mu \mathrm{m}, 1.35-$ $1.45 \mu \mathrm{m}, 1.8-2.0 \mu \mathrm{m}$ and longward of $2.4 \mu \mathrm{m}$. We do not detect neither the two KI doublets near 1.18 and $1.24 \mu \mathrm{m}$, nor the $\mathrm{NaI}$ doublet near $1.14 \mu \mathrm{m}$, but this is certainly due to the lower SNR of the spectrum in the $J$ band. We possibly detect the NaI doublet in absorption near $2.2 \mu \mathrm{m}$ and some $\mathrm{H}_{2}$ lines in emission $(1.96,2.12,2.22$ and around $2.4 \mu \mathrm{m}$ ). The $\mathrm{H}_{2}$ emission is the signature of a very young object, it can be caused by shocked gas in a molecular outflow or by UV excitation of gas in a circumstellar disk. Finally, we clearly detect the $\operatorname{Br} \gamma$ line in emission. Muzerolle et al. (1998) have found a tight correlation in $\mathrm{T}$ Tauri stars between the strength of the $\mathrm{Br} \gamma$ line and the accretion luminosity as measured from the hot continuum excess. This could suggest that BD-Ser 1 is accreting matter from a circumstellar disk.

To estimate the luminosity of the object, we used the Leggett et al. (2001) procedure. We have integrated the observed flux, adding an estimated contribution at shorter wavelength with a linear extrapolation to zero flux at zero wavelength, and a contribution at wavelengths beyond $2.5 \mu \mathrm{m}$, estimated assuming a Rayleigh-Jeans tail. The total flux obtained is $F_{\mathrm{T}}=8.1 \times 10^{-15} \mathrm{~W} \mathrm{~m}^{-2}$, and the total luminosity of the star is $L_{\mathrm{T}}=1.69 \times 10^{-2} L_{\odot}$. This is very bright when compared to the usual observed luminosity of early L-type, few hundreds Myr old brown dwarfs

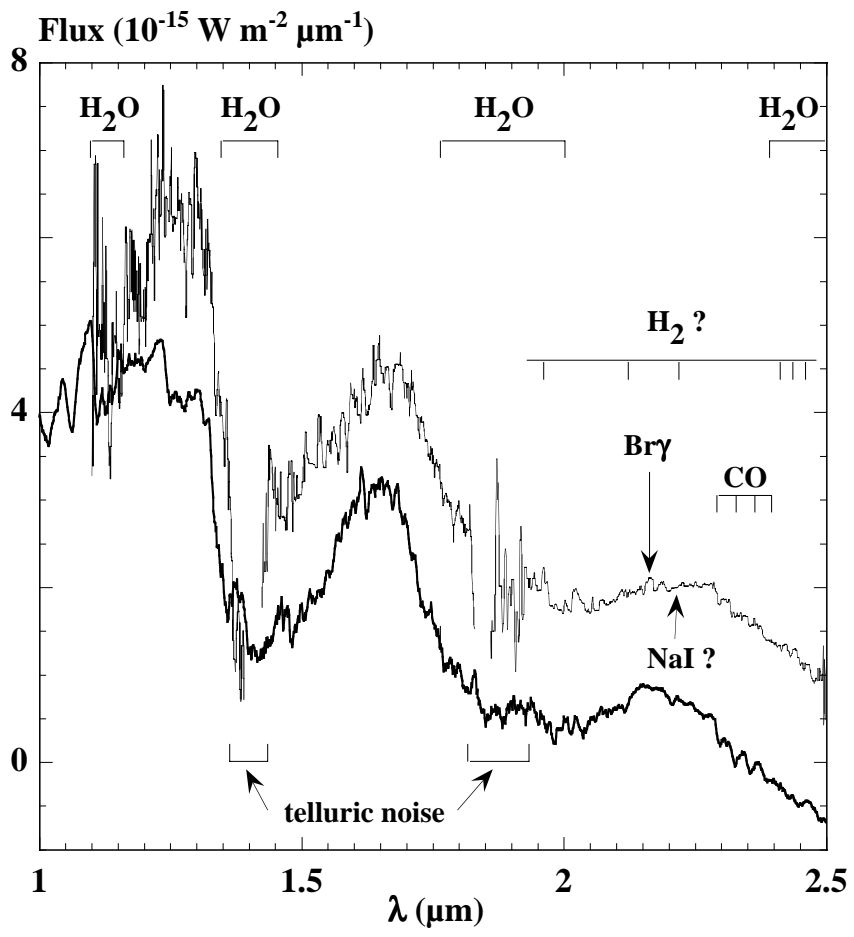

Fig. 2. Dereddened spectrum of BD-Ser 1 obtained with VLT/ISAAC, smoothed to a spectral resolution of $75 \AA$ (thin line), compared to a theoretical spectrum of a $\sim 3.5 \mathrm{Myr}$, $0.05 M_{\odot}$ brown dwarf (see text) smoothed to a similar spectral resolution and y-shifted by $-1.5 \times 10^{-15}$ for clarity (thick line).

$\left(\sim 10^{-4} L_{\odot}\right.$, Reid et al. 2001), but this is expected from brown dwarf evolutionnary tracks. For instance, a $0.05 M_{\odot}$ brown dwarf is more than 2 orders of magnitude brighter at $1 \mathrm{Myr}$ than at $500 \mathrm{Myr}$ (Baraffe et al. 1998).

We have used the theoretical spectra of Allard et al. (2001) to confirm the physical parameters of BD-Ser 1 and reduce their uncertainties. Figure 2 shows our observed spectrum, and a nextgen model computed for $T_{\text {eff }}=2800 \mathrm{~K}$ and $\log g=4$, the closest physical parameters of those of a $0.05 M_{\odot}, 3.5 \mathrm{Myr}$ old brown dwarf $\left(T_{\text {eff }}=2850 \mathrm{~K}, \log g=3.75, L=1.48 \times 10^{-2} L_{\odot}\right.$, Baraffe et al. 1998). The agreement between the two spectra is very good. In order to assess the young brown dwarf status of BD-Ser 1, we have also considered a theoretical spectrum of an older object at the hydrogen burning limit $\left(10 \mathrm{Myr}, 0.075 M_{\odot}\right)$. This last spectrum differs significantly from the observed one and we conclude that BD-Ser 1 is a $0.05 \pm 0.02 M_{\odot}$ brown dwarf of $3.5 \pm 2 \mathrm{Myr}$. This age is consistent with the conservative value estimated by Kaas (1999) for the Serpens cluster. Together with its visual absorption $A_{V}=10.7$, this is a strong evidence that BD-Ser 1 is physically associated with the cloud and was formed within, at the same location and time than other young stars. 
Table 3. NIR indices for BD-Ser 1 and corresponding spectral type (ST). Letters in brackets refer to the references given in the text.

\begin{tabular}{ccccc}
\hline Index & & $\begin{array}{c}\text { BD-Ser 1 } \\
\text { value }\end{array}$ & $\begin{array}{c}\text { BD-Ser 1 } \\
\text { ST }\end{array}$ & $\begin{array}{c}\text { Model } \\
\text { ST }\end{array}$ \\
\hline $\mathrm{K} 1$ & {$[\mathrm{a}]$} & 0.07 & $\mathrm{~L} 0$ & $\mathrm{~L} 1$ \\
$\mathrm{~K} 2$ & {$[\mathrm{a}]$} & 0.02 & $\mathrm{~L} 2$ & $\mathrm{~L} 1$ \\
$\mathrm{H}_{2} \mathrm{O}^{\mathrm{A}}$ & {$[\mathrm{b}]$} & 0.68 & $\mathrm{~L} 1$ & $\mathrm{~L} 1$ \\
$\mathrm{H}_{2} \mathrm{O}^{\mathrm{B}}$ & {$[\mathrm{b}]$} & 0.75 & $\mathrm{~L} 2$ & $\mathrm{~L} 3$ \\
$\mathrm{H}_{2} \mathrm{O}^{\mathrm{C}}$ & {$[\mathrm{b}]$} & 0.65 & $\mathrm{~L} 1-\mathrm{L} 2$ & $\mathrm{~L} 3$ \\
$\mathrm{H}_{2} \mathrm{O}^{\mathrm{D}}$ & {$[\mathrm{b}]$} & 0.86 & $\mathrm{~L} 0$ & $\mathrm{~L} 1$ \\
$\mathrm{sHJ}$ & {$[\mathrm{c}]$} & 0.35 & $\mathrm{~L} 0$ & $\mathrm{~L} 2-\mathrm{L} 3$ \\
$\mathrm{sKJ}$ & {$[\mathrm{c}]$} & 1.03 & $\leq \mathrm{L} 0$ & $\mathrm{~L} 0$ \\
$\mathrm{sH}_{2} \mathrm{O}^{\mathrm{J}}$ & {$[\mathrm{c}]$} & 0.18 & $\mathrm{~L} 2-\mathrm{L} 3$ & $\mathrm{~L} 0$ \\
$\mathrm{sH}_{2} \mathrm{O}^{\mathrm{H} 1}$ & {$[\mathrm{c}]$} & 0.37 & $\mathrm{~L} 2$ & $\mathrm{~L} 2$ \\
$\mathrm{sH}_{2} \mathrm{O}^{\mathrm{H} 2}$ & {$[\mathrm{c}]$} & 0.41 & $\mathrm{~L} 1-\mathrm{L} 2$ & $\mathrm{~L} 4-\mathrm{L} 5$ \\
$\mathrm{sH}_{2} \mathrm{O}^{\mathrm{K}}$ & {$[\mathrm{c}]$} & 0.04 & $\leq \mathrm{L} 0$ & $\mathrm{~L} 1$ \\
$\mathrm{H}_{2} \mathrm{O} 1.5 \mu \mathrm{m}$ & {$[\mathrm{d}]$} & 1.26 & $\mathrm{~L} 0$ & $\mathrm{~L} 1$ \\
$\mathrm{CH}_{4} 2.2 \mu \mathrm{m}$ & {$[\mathrm{d}]$} & 0.93 & $\mathrm{~L} 3$ & $\mathrm{~L} 4$ \\
$Q_{H}-$ band & {$[\mathrm{e}]$} & 0.31 & $\mathrm{~L} 3$ & $\mathrm{~L} 4$ \\
\hline
\end{tabular}

\subsection{Spectral type}

Numerous recent studies have linked near-infrared and far-red/optical indices to give a framework for brown dwarf spectral type classification (Tokunaga \& Kobayashi 1999 [a]; Reid et al. 2001 [b]; Testi et al. 2001 [c]; Geballe et al. 2001 [d] and Martin 2000 [e]). These indices, computed for BD-Ser 1, and the corresponding spectral types, are reported in Table 3, together with the spectral types derived from the model spectra of a $0.05 M_{\odot}, 3.5 \mathrm{Myr}$ old brown dwarf. All the indices but two give a result consistent with a brown dwarf of spectral type L0-L3, in agreement with those obtained from the theoretical spectrum. Further investigations (both observational and theoretical) are needed to investigate the reasons why the sKJ and $\mathrm{sH}_{2} \mathrm{O}^{\mathrm{K}}$ indices correspond to earlier spectral types, but it is most likely that BD-Ser 1 is a young brown dwarf of spectral type L0-L3. Moreover, we can also use the Allard et al. (2001) theoretical spectra to compute the indices for the same brown dwarf with an age of $200 \mathrm{Myr}$. We find that most of these indices are consistent with a spectral type later than L9, hence BD-Ser 1 is a future $\mathrm{T}$ type brown dwarf.

\section{Summary}

We have performed a deep near-infrared survey images of a 50 square arcmin region in the Serpens cloud with SOFI on the NTT. This survey presents an unprecedented detection limit of $M_{K}=18.6$, deep enough to probe the substellar domain of the observed field despite its high internal extinction. Among thousands of stars detected,
20 brown dwarf candidates were selected for follow-up near-infrared spectroscopic observations performed using ISAAC at the VLT. These observations allowed to confirm the brown dwarf status of at least one of our candidates. According to evolutionary tracks for brown dwarfs, we estimate the age of this object to be $3.5 \pm 2 \mathrm{Myr}$ and its mass to be $0.05 \pm 0.02 M_{\odot}$. From its NIR indices, we estimate its present spectral type to be L0-L3, and from the model, its future spectral type (at $t \geq 200 \mathrm{Myr}$ ) to be $T$. This is the first young brown dwarf ever found deeply embedded in a star formation region.

Acknowledgements. The authors aknowledge enriching discussion with F. Allard and X. Delfosse in the course of writing this letter. We also thank the ESO observatories and the NTT and VLT staff for their help during the observations. This research has made use of NASA's Astrophysics Data System Bibliographic Services.

\section{References}

Allard, F., Hauschildt, P. H., Alexander, D. R., et al. 2001, ApJ, 556, 357

Baraffe, I., Chabrier, G., Allard, F., \& Hauschildt, P. H. 1998, A\&A, 337, 403

Basri, G. 2000, ARA\&A, 38, 485

Béjar, V. J. S., Martin, E. L., Zapatero Osorio, M. R., et al. 2001, ApJ, in press

Devillard, N. 1999, ADASS VIII, ASP Conf. Ser., 172, 333

Devillard, N. 2001, ADASS X, ASP Conf. Ser., 238, 525

Eiroa, C., \& Casali, M. M. 1992, A\&A, 262, 468

Geballe, T. R., Knapp, G. R., Leggett, S. K., et al. 2001, ApJ, in press

Giovannetti, P., Caux, E., Nadeau, D., \& Monin, J.-L. 1998, A\&A, 330, 990

Kaas, A. A. 1999, AJ, 118, 558

Leggett, S. K., Allard, F., Geballe, T. R., et al. 2001, ApJ, 548, 908

Lodieu, N., et al. 2002b, A\&A, in preparation

Lucas, P. W., \& Roche, F. P. 2001, MNRAS, 314, 858

Lucas, P. W., Roche, F. P., Allard, F., \& Hauschildt, P. H. 2001, MNRAS, 326, 695

Martin, E. L. 2000, the Ultracool Dwarfs Workshop, IAU General Assembly, Manchester, ed. H. Jones, \& I. Steele (Springer Verlag)

Martin, E. L., Zapatero Osorio, M. R., Barrado y Navascuès, D., et al. 2001a, ApJ, 558, L117

Martin, E. L., Dougados, C., Magnier, E., et al. 2001b, ApJ, 561, L195

Moorwood, A. F. M., \& Spyromilio, J. 1997, in The Early Universe with the VLT, ed. J. Bergeron (Springer), 21

Muzerolle, J., Hartmann, L., \& Calvet, N. 1998, AJ, 116, 2965

Persson, S. E., Murphy, D. C., Krzeminski, W., et al. 1998, AJ, 116,2475

Pickles, A. J. 1998, PASP, 110, 863

Reid, I. N., Burgasser, A. J., Cruz, K. L., et al. 2001, AJ, 121, 1710

Straizys, V., Ernis, K., \& Bartait, S. 1996, Baltic Astron., 5, 125

Testi, L., D'Antona, F., Ghinassi, F., et al. 2001, ApJ, 552, L147

Tokunaga, A., \& Koyabashi, N. 1999, AJ, 117, 1010 